\title{
Homemade Infant Warmer
}

\author{
${ }^{1}$ Dr Shankar Ram HS, ${ }^{2}$ Dr Sandhya Ram DA \\ ${ }^{I}$ MS FMAS DMAS FAGE .Consultant Surgeon. Sandhyaram Hospital Palakkad Kerala -India \\ ${ }^{2}$ MBBS DA . Anesthesiologist.Sandhya Ram Hospital .Kadampazhipuram .Palakkad Kerala - India
}

\section{Introduction}

Maintenance of Temperature and humidity is crucial in the care of the new born. The requirements vary depending on the gestational age at which the baby is born. A humidity of 60 to 80 percent is essential. $(1,2)$.Even after the discharge of the baby from the hospital the new born is subjected to diurnal variations in temprature and cold at night . Using low cost available materials we have developed a cost effective neonatal open care system suitable for tropical weather in south Indian population. The temperature varies from 38 degrees $\mathrm{C}$ in day in summer to 16 or 18 degree $\mathrm{C}$ in the night at winter. The baby is at risk of hypothermia in winters or exposure to low temperatures when there are heavy rains.

Electric bulbs were used as source of heat energy in creating warm environment for the care of piglets and showed better results as compared to control (3).This sounds economical and feasible to be applied to newborn especially in low resource setting. The basic design of the device comprises 200 watt electric bulb with protective covering or mesh, a plastic Trolley ,a bulb holder , a cardboard or plywood. An Electric switch with regulator and digital Clock with temperature recorder in degree centigrade. Washed and wet blankets are spread or hung on the side of the walls which increases the humidity of the room.

\section{Design}

Distance between bulb and baby is maintained between 23 to 25 inches by adjusting interlocking screws in PVC pipe that holds the bulb holder. .The intensity of light is adjusted by rotating regulator which in turn adjusts the temperature .Initial warming takes 5 to 0 minutes depending on room temperature. Maximum surface temp obtained with this setting is 33 degrees when outside temp is 18 degrees at the specified height of the bulb.

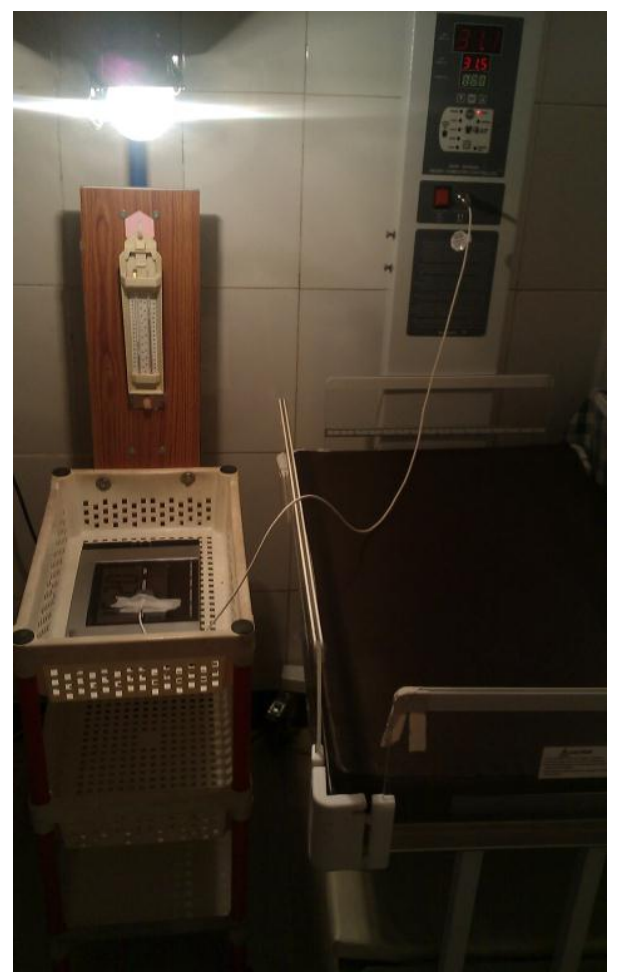

Figure 2 : Comparison of temperature conditions with infant warmer . 


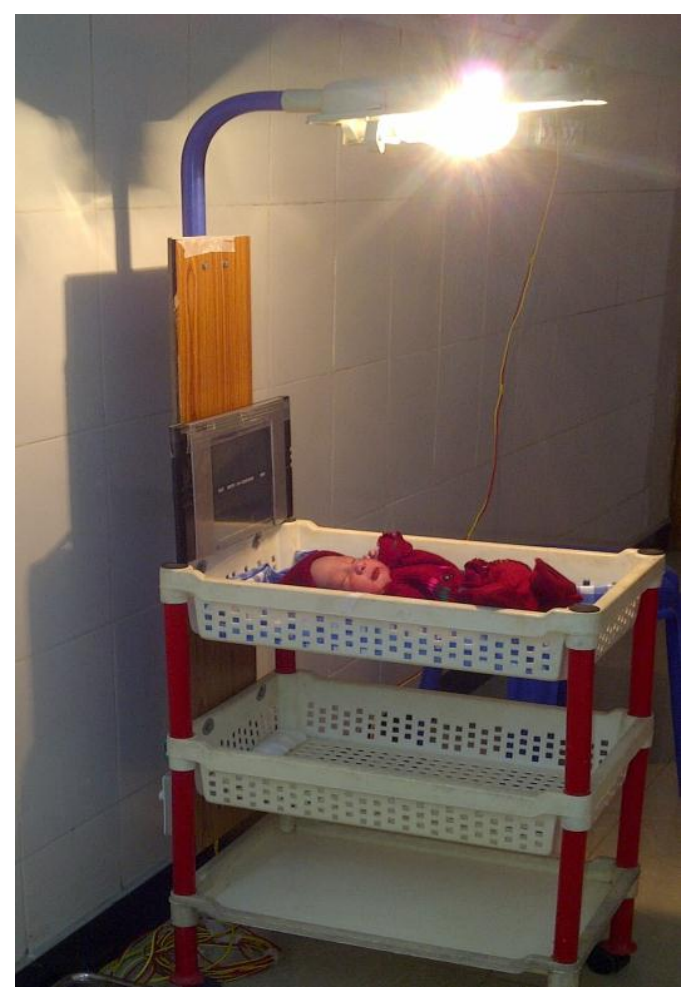

Figure 2: Custom made infant warmer for care of new born. The bulb regulator is fitted in the lower compartment or at convenient place for adjustment.

Basic aim was to keep baby warm which is amenable for home care. Baby is wrapped in clean towel within which ,temperature of the baby's axilla is monitored with digital thermometer .It is usually between 1 to 2 degrees $\mathrm{C}$ higher than the surface temperature. Bulb needs to be changed when higher temperatures are recorded beyond 34 degrees. Eyes are covered with Head cap like a sunshade which also prevents loss temperature from the scalp and prevents direct exposure of the light .Cochrane review shows after 32 weeks there is less damage to eyes on exposure to light however still necessary precautions are taken (4).

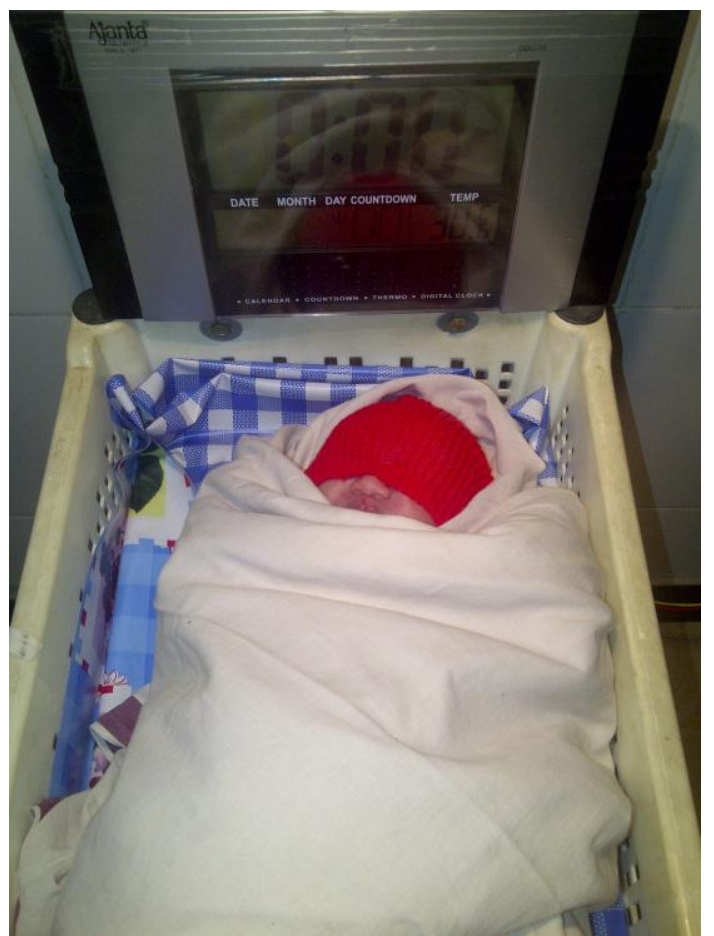

Figure 3: Temperature measurements are taken from the digital reading and the light intensity is adjusted accordingly to the desired temperature. 


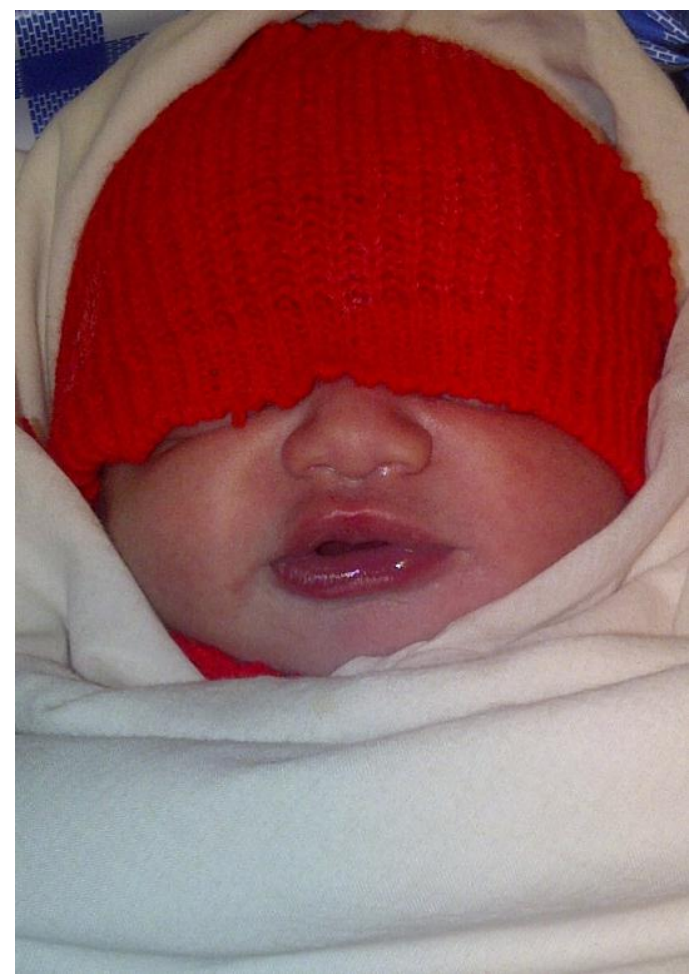

Figure 4: Eyes are covered with extension of head cap.

\section{Discussion}

Babies born with mature liquor [ mean amniotic fluid turbidity /optical density at normal labor is around 0.98 at $650 \mathrm{~nm}$ ] (5) after 36 weeks gestation have well developed thermoregulatory mechanisms and require lesser warmth as compared to earlier gestation. Theoretically only 2 percent of electricity is transmitted as light and hence the tungsten bulb is more efficient source of heat rather than light .Here we have used electric bulb of 200 wattage as source of heat for the care of new born who were mature and also which were shifted out of nursery and were fit for discharge. Invitro prototype testing with infant warmer and concurrent comparison of temperature recordings showed satisfying results and later babies were nursed. (Figure 1 and 2 ). It is quite useful when mother requires isolation and rooming in cannot be done. There is no chance of over heat as the maximum temperature is fixed for a particular adjusted height of the bulb. The device requires personal monitoring as there is no alarm or probes and also keeping the possibility of voltage fluctuations which can corrected by a stabilizer. However there is no risk of skin temperature probe displacement and over warming.

\section{References}

[1]. British Columbia reproductive care program .Neonatal Thermoegulation.Newborn Guideline 2.

[2]. Knobel, Robin; Holditch-Davis, Diane. Thermoregulation and Heat Loss Prevention After Birth and During Neonatal IntensiveCare Unit Stabilization of Extremely Low-Birthweight Infants.Advances in Neonatal Care: October 2010 - Volume 10 - Issue - p S7-S14

[3]. B O Ogunbameru, E T Kornegay and C M Wood. Evaluation of methods of providing supplemental heat to newborn pigs during and after farrowing. J Anin Sci 1991, 69:3939-3944.

[4]. Jorge EC, Jorge EN, El Dib RP. Early light reduction for preventing retinopathy of prematurity in very low birth weight infants. Cochrane Database of Systematic Reviews 2013, Issue 8. Art. No.: CD000122. DOI: 10.1002/14651858.CD000122.

[5] SamarthaRam H, Shankar Ram SR .Sandhya S. Individual Term for each fetus: with surge in amniotic fluid optical density. BJOG :121(s2):EP7.99 